\title{
Estrategia de calidad del servicio para mejorar la atención al cliente en heladería
}

\author{
Service quality strategy to improve customer service in ice cream parlor
}

Estratégia de qualidade de serviço para melhorar o atendimento ao cliente em sorveteria

Recibido: enero 2021

Arbitrado: febrero 2021

Aceptado: marzo 2021

Publicado: mayo 2021

\author{
Ana Edith Otalora Gutiérrez \\ anaedithotalora@outlook.es \\ https://orcid.org/0000-0002-1002-9777 \\ Universidad Universidad Privada “Domingo Savio”, Potosí-Bolivia
}

RESUMEN

El objetivo de la investigación fue elaborar una estrategia de calidad del servicio para mejorar la atención al cliente en la heladería "El Estudiante". La metodología fue de tipo descriptiva y propositiva, con un enfoque cuantitativo y cualitativo; los métodos usados fueron; análisis síntesis, inductivo deductivo, histórico lógico y comparativo. Las técnicas e instrumentos usados fueron, observación, entrevista y encuesta. Se obtuvo como resultado, que existen falencias y causales que ocasionan que no se brinde una buena atención al cliente. Se concluye, que con la propuesta se implementaran herramientas de calidad de servicio por lo que fue, desarrollada en base a la confiabilidad, sensibilidad, seguridad, y empatía. Cabe destacar que, esta estrategia debe ser empleada de forma conjunta, es decir, gerencia y personal para conseguir la atención al cliente ideal para la heladería "El Estudiante".

Palabras clave: Calidad de servicio; atención al cliente; dimensiones; heladería, Bolivia
ABSTRACT

The objective of the research was to develop a service quality strategy to improve customer service at the ice cream parlor "El Student". The methodology was descriptive and purposeful, with a quantitative and qualitative approach; the methods used were; synthesis, inductive deductive, logical and comparative historical analysis. The techniques and instruments used were, observation, interview and survey. It was obtained as a result, that there are shortcomings and causes that cause good customer service not to be provided. It is concluded that with the proposal, quality of service tools will be implemented for what it was, developed based on reliability, sensitivity, security, and empathy. It should be noted that this strategy must be used jointly, that is, management and staff to achieve the ideal customer service for the ice cream parlor "El Student".

Palabras clave: Quality of service; customer support; dimensions; ice cream shop, Bolivia

\section{RESUMO}

O objetivo da pesquisa foi desenvolver uma estratégia de qualidade de serviço para melhorar o atendimento ao cliente da sorveteria "El estudante". A metodologia foi descritiva e proposital, com abordagem quantitativa e qualitativa; os métodos usados foram; síntese, análise histórica indutiva dedutiva, lógica e comparativa. As técnicas e instrumentos utilizados foram observação, entrevista e inquérito. Obteve-se como resultado, que existem lacunas e causas que fazem com que não seja prestado um bom serviço ao cliente. Conclui-se que com a proposta serão implementadas ferramentas de qualidade de serviço, para as quais foi desenvolvida com base na confiabilidade, sensibilidade, segurança e empatia. É importante ressaltar que esta estratégia deve ser utilizada em conjunto, ou seja, gestão e equipe para conseguir o atendimento ideal para a sorveteria "El estudante".

Palabras clave: Qualidade de serviço; Suporte ao cliente; dimensões; sorveteria, Bolívia 


\section{INTRODUCCIÓN}

$\mathrm{E}$ I marketing funciona como un paso para definir el eslabón de la comercialización de los productos y servicios que son promocionados por una empresa u organización por ello, Kotler y Armstrong (2008), lo definen como el proceso a través del cual las empresas crean valores para los clientes con la finalidad de establecer relaciones sólidas que garantice la obtención del cambio en el valor de los clientes. También, este es divido en varios tipos que sirven para diferentes situaciones, necesidades, canales, empresas y consumidores a medida estos se van presentando.

En este sentido, se ingresa específicamente al marketing de servicios con su principal elemento diferenciador que radica en la intangibilidad que, si bien va dirigido a una categoría especial de productos que son los servicios, tales como educación, transporte, diversión, créditos, etc., también se da muchas ofertas de mercado que se componen de una combinación mixta de bienes y servicios, por ejemplo, en un restaurante de comida rápida, el cliente consume tanto el bien como el servicio. Cabe destacar que, en el marketing de servicios cobran vital importancia las personas, que dan un valor añadido al servicio, siendo esta la atención al cliente.

Por consiguiente, se tiene el hecho de que la experiencia del cliente está estrechamente ligada al éxito o al fracaso de una empresa; esta interacción influye decisivamente en él, en su opinión acerca de la empresa, en la opinión que transmita a los demás, y en su decisión de seguir o no contando con esta. Dentro de la relación que el usuario establece con la organización, la atención al cliente es una parte esencial, pues es el único momento en el que el cliente tiene contacto con la empresa.

La atención al cliente es de vital importancia para las empresas, debido a que se trata de superar las expectativas de los clientes con acciones por parte de los empleados, tales que son expresadas desde el ingreso del cliente con un saludo de bienvenida, sonreír, ofrecer muestras gratis, establecer contacto visual, preguntar con entusiasmo las inquietudes, usar buenas habilidades para escuchar y dar las gracias por la compra, tratar al cliente como si fuera un amigo, vecino o invitado. Esto permite la personalización de la atención a los clientes que los hace sentirse especiales puesto que él es el que valora la calidad en la atención que recibe.

En este sentido, Ongallo (2007), define la atención al cliente como un servicio, de calidad de algo en donde no se puede pesar, ni medir, pero que a largo plazo deja un regusto en el cliente como algo bueno, regular o malo, a esto se le conoce como la parte intangible ya que se encuentra inextricablemente unida a la figura del vendedor.

La American Marketing Association (como se citó en Kotler y Keller, 2012) ofrece la siguiente definición formal sobre lo que ellos consideran como Marketing considerándole como la actividad o grupo de entidades y procedimientos para crear, comunicar, entregar e intercambiar ofertas que se consideren de valor para los consumidores, clientes, socios y la sociedad en general. 
El marketing no es solo vender y anunciar a través de la publicidad como muchas personas lo consideran, sino que va más allá, siendo un conjunto de acciones que busca identificar y satisfacer las necesidades del cliente para crear relaciones con ellos.

El marketing va evolucionando para adaptarse a los cambios que van ocurriendo en la sociedad algunos tipos de marketing que menciona Kotler y Keller (2012), son Marketing de productos, Marketing de servicios, Marketing directo, Marketing interactivo, Marketing interno, Marketing externo y Marketing de relaciones

Según Thompson (2008), es una rama del marketing que se especializa en una categoría especial de productos, los servicios, los cuales, apuntan a satisfacer ciertas necesidades o deseos del mercado, tales como educación, transporte, protección, jubilación privada, asesoramiento, diversión, créditos, etc.

En consecuencia, el marketing de servicios debe entenderse como una ampliación del concepto tradicional de marketing, en el que se reafirma los procesos de intercambio entre compradores y empresas con el objetivo final de satisfacer las necesidades de los clientes, pero en función de las características específicas de los servicios, es por este entendido que se adopta la postura de Thompson.

En el Diccionario de Marketing (1999), es definido como el cliente o persona u organización que realiza una compra. Puede estar comprando en su nombre, y disfrutar personalmente del bien adquirido, o comprar para otro. Resulta la parte de la población más importante de la compañía.

En este orden de ideas, la atención al cliente según Horovitz (1991), sería un conjunto de actividades desarrolladas en una organización con la finalidad de orientarla al mercado, al mismo tiempo encaminadas a identificar las necesidades de los clientes en la compra para satisfacerlas, logrando de este modo cumplir sus expectativas, y, por tanto, crear o incrementar la satisfacción de los clientes.

A su vez, para Horovitz (1991), la atención al cliente constituye a la prestación que el cliente espera como consecuencia de la imagen, el precio y la reputación del producto o servicio que recibe.

Aunado a ello, es relevante determinar que la atención al cliente se refleja como las actividades que realiza la empresa para satisfacer las necesidades de los clientes, a través de la comunicación e interacción directa con ellos.

La atención al cliente ha venido tomando fuerza acorde al aumento de la competencia, ya que mientras más exista, los clientes tienen mayor oportunidad de decidir en donde adquirir el producto o servicio que están requiriendo, es aquí donde radica dicha importancia de irlo perfeccionando y adecuando a las necesidades de los clientes, ya que estos mismo son quienes tendrán la última palabra para decidir.

La importancia se guía por los siguientes aspectos, mencionados por López (2013), la competencia es cada vez mayor, por ende, los productos ofertados aumentan notablemente y son más variados, por lo que se hace necesario ofrecer un valor agregado; también, los competidores se van equiparando en calidad y precio, por lo que se hace necesario buscar una diferenciación; y los clientes son cada vez más exigentes, ya no sólo buscan precio y calidad, sino también, una buena atención, un ambiente agradable, comodidad, un trato personalizado, un servicio rápido. 


\section{La comunicación en la atención al cliente}

Para Paz (2007), en la atención al cliente, la empresa se comunica con sus clientes a través de su personal. Aunque en la era de las telecomunicaciones, la mayor parte de los contactos se siguen dando en persona o por teléfono, aunque cada vez hay más empresas que añaden a estos canales tradicionales la venta a través de internet, de catálogo venta por correo, etc. Por lo que es importante que cuando la empresa se comunique con sus clientes, mediante la palabra hablada, no sólo transmite la información concreta relativa a la situación y producto específico, ya que toda empresa que desee permanecer en el mercado necesita tener clientes fieles, es en la empresa donde la comunicación adquiere especial relevancia.

En la atención al cliente se trata básicamente de la palabra, que puede ser oral o escrita y que tendrá unos significados conocidos y compartidos por ambos interlocutores para que dicha comunicación llegue a buen fin.

Existen varias formas de clasificar a los clientes, sin embargo, para este estudio se toma en cuenta la tipología que comprende a los clientes según su personalidad y la manera de tratarlos. Para Palomo (2014, p. 74), los clientes se clasifican de la siguiente manera:

Cliente sabelotodo. Cree saberlo todo, mantiene una actitud de superioridad, es muy exigente y retado, en ocasionas suele mostrarse agresivo. Se debe mostrar una solución de manera que no se ofenda, interesarse por su conocimiento, hágale ver que sus aportes fueron valiosos para la solución y tomar una actitud serena y atenta, pero no dejarse dominar.

Cliente minucioso. Sabe muy bien lo que quiere, utiliza pocas palabras, exige respuestas concretas y va directo al grano, utiliza pocas palabras. Para este tipo de clientes se debe brindar una atención rápida, las respuestas deben ser precisas, demostrar seguridad y seriedad, la cortesía y amabilidad son vitales, también demostrar interés en sus necesidades.

Cliente indeciso. Es tímido e inseguro, le cuesta decidirse, teme plantear claramente que cosa es lo que quiere; necesita reflexionar antes de hacer la compra, a veces reflexiona demasiado; generalmente no compra de inmediato. Es el típico "Regreso después". Para tratarlo anímale a que exprese sus necesidades, sonrisa amplia y demostrar seguridad en el producto, conocer la competencia y puntos fuertes sobre ellos y aceptar sus puntos de vista.

La calidad del servicio se puede plantear como una situación en la cual, una empresa otorga calidad y servicios superiores a sus clientes, propietarios y empleados, lo que Significa que todas las características, actos e información deben aumentar la capacidad para producir valor para el cliente (Tigani, 2006).

Según Pizzo, (citado por López, 2013) dice que la calidad del servicio Es el hábito desarrollado y practicado dentro de una organización a fin de interpretar las necesidades y expectativas de los clientes y ofrecerles, en consecuencia, un servicio accesible, adecuado, ágil, flexible, apreciable, útil, oportuno, seguro y confiable, aún bajo situaciones imprevistas o ante errores, de tal manera que el cliente se sienta comprendido, atendido y servido personalmente, con dedicación y eficacia, y sorprendido con mayor valor al esperado, proporcionando en consecuencia mayores ingresos y menores costos para la organización.

La Calidad del Servicio es la capacidad no sólo para satisfacer, sino también para superar las expectativas del cliente. 
La investigación sugiere que los clientes no perciben la calidad en una forma unidimensional, sino más bien juzgan la calidad con base en múltiples factores relevantes para el contexto.

Según Zeithaml et al. (2009), son confiabilidad (entrega las promesas), sensibilidad (estar dispuesto ayudar), seguridad (inspirar credibilidad y confianza), empatía (tratar a los clientes como individuos), tangibles (representar al servicio físicamente).

La escala SERVQUAL es una escala multidimensional para captar las percepciones y expectativas de la calidad del servicio por parte del cliente. La escala SERVQUAL se publicó por primera vez en 1988 y ha experimentado en las numerosas mejoras y revisiones desde entonces. En la actualidad la escala contiene 22 reactivos de percepción que están distribuidos a lo largo de las cinco dimensiones de calidad del servicio (Zeithaml et al., 2009).

Tabla 1. Escala SERVQUAL.

\section{PERCEPCIONES}

\section{Reactivos en la dimensión de confiabilidad}

1. Cuando la compañía XYZ promete hacer algo en un cierto tiempo, lo cumple

2. Cuando el cliente tiene un problema, la compañía XYZ muestra un sincero interés en resolverlo.

3. La compañía $X Y Z$ desempeña bien el servicio la primera vez.

4. La compañía XYZ proporciona sus servicios en el momento en el que promete hacerlo.

5. La compañía XYZ insiste en registros libres de error.

\section{Reactivos en la dimensión de sensibilidad}

1. La compañía XYZ mantiene informado a los clientes con respecto a cuándo se ejecutarán los servicios.

2. Los empleados en la compañía XYZ dan al cliente un servicio expedito.

3. Los empleados en la compañía XYZ siempre están dispuesto a ayudar al cliente.

4. Los empleados en la compañía XYZ nunca están demasiado ocupados para responder una solicitud del cliente.

\section{Reactivos en la dimensión de seguridad}

1. La compañía $X Y Z$ tienen empleados que dan atención personalizada a los clientes.

2. La compañía $X Y Z$ se ocupa de sus mejores intereses

3. Los empleados de la compañía XYZ entienden las necesidades específicas del cliente.

4. La compañía XYZ tiene horarios de operación convenientes para todos sus clientes.

\section{Reactivos en la dimensión de empatía}

1. La compañía XYZ le da atención individual al cliente.

2. La compañía $X Y Z$ tienen empleados que dan atención personalizada a los clientes.

3. La compañía $X Y Z$ se ocupa de sus mejores intereses

4. Los empleados de la compañía $X Y Z$ entienden las necesidades específicas del cliente.

5. La compañía XYZ tiene horarios de operación convenientes para todos sus clientes.

\section{Reactivos en la dimensión de tangibles}

1. La compañía $X Y Z$ tiene equipo de aspecto moderno.

2. Las instalaciones físicas de la compañía $X Y Z$ son visualmente atractivas.

3. Los empleados de la compañía XYZ se ven pulcros.

4. Los materiales asociados con el servicio (como panfletos o decoraciones) son visualmente atractivos en la compañía XYZ.

Nota: Adaptado de “Marketing de Servicios”, de Zeithaml, V., Bitner, M. y Gremler, D., 2009, pp. 152-153, México: McGraw-HiII/Interamericana Editores, S.A. de C.V. 
La calidad del servicio es muy importante para la empresa para poder mejorar la atención al cliente, es así que esta escala SERQUAL ayudará a que pueda ejecutarse de una mejor manera y tener mejores resultados.

Una buena atención al usuario puede marcar la diferencia entre un cliente satisfecho y un cliente que no lo está, crea buena voluntad, relaciones positivas con los clientes y lo importante es que agrandan las ventas. Si todo el mundo va a hablar de la atención al cliente que presta tu empresa, ¿no será mejor que hablen bien? Algunos estudios descubrieron que la gente que se siente bien durante la experiencia de compra tiene tendencia a comprar más tiempo y más productos.

En Bolivia este tipo de establecimientos va acorde a la región en donde se encuentre, tal el caso de las heladerías Brosso, Globos, Dumbo, Frutigur entre otros, que viéndose instauradas en las ciudades de mayor crecimiento del país como; La Paz, Santa Cruz y Cochabamba en Bolivia, reflejan en ellas años en el mercado, un gran capital e infraestructura propicia, destacando el ofrecimiento en valor añadido que se ve en sus servicios.

En la ciudad de Potosí, en Bolivia la heladería “El Estudiante” surge el año 1986, a idea de la Sra. Mercedes Alcaraz Benavidez. En el año 1998 se forma una sociedad con la Sra. Rosario Miranda. Viendo la necesidad de que la población respondió positivamente al servicio, se la reubicó en la calle Bustillo n¹036, lugar donde funciona actualmente.

Hoy en día la heladería es aceptada por la población potosina, siendo su principal labor brindar un servicio con un producto de calidad con helados artesanales, al gusto del cliente. Sin embargo, se presenta en ocasiones que, en la ciudad de Potosí, la atención al cliente no sea uno de los servicios de los que más se cuida en las empresas, estas caen en el error de no prestarle el interés que se merece, viéndose involucrada también en este problema la heladería "El Estudiante"; siendo que esta empresa ya cuenta con una trascendencia en el mercado, se evidencia que existe una atención al cliente inadecuado, en el que a la hora de consumir el producto existen largas esperas, lo que genera molestia en los clientes por la lentitud en las tareas realizadas.

Es por ello que, los clientes no se sentían especiales al ser atendidos, la relación que se generaba era áspera sin la existencia de un vínculo de amistad y confianza que lógrese cumplir e incluso superar las expectativas del cliente. En este sentido esta problemática, radica en la atención en la prestación del servicio, por lo es importante considerar un cambios que permitan una mejor atención a los clientes que frecuentan heladería, a fin de establecer excelente atención al cliente y así, asegurar el incremento de las ventas; para ello, que el estudio plantea como objetivo elaborar una estrategia de calidad del servicio para mejorar la atención al cliente en la heladería "El Estudiante" de la ciudad de Potosí en Bolivia.

El propósito de la investigación constituye el hecho de mitigar las falencias tales como la ineficiencia en atención al cliente que atraviesa la empresa. Lo cual traerá beneficios como la fidelización, satisfacción del cliente, creación de un vínculo comercial más sólido con los clientes de cubrir sus expectativas, y, por ende, aumento en sus utilidades económicas, fundamental no sólo para el éxito de la empresa, sino también, para la supervivencia comercial de la misma, por su parte, los clientes se sentirán especiales, bien atendidos y contentos con la experiencia de compra. 


\section{MÉTODO}

$\mathrm{L}$

a investigación se desarrolló con una metodología de tipo descriptiva, puesto que se describió y caracterizó con precisión los sucesos, hechos y situaciones que se desarrollaron en la actualidad en cuanto a la atención al cliente que se tiene en la heladería “El Estudiante”, y propositiva, ya que, se planteó una propuesta de calidad del servicio para superar la problemática actual y las deficiencias encontradas.

El diseño de la investigación fue documental, con la finalidad de recopilar información teórica y obtener datos a partir de diversas fuentes documentales que abordan la temática, que sirvieron de soporte para la elaboración del estudio y conocimiento profundo de las variables, y de campo, ya que, se realizó recolectando datos de fuentes primarias, es decir, directamente de los involucrados que son los clientes, trabajadores y gerente de la heladería "El Estudiante".

A su vez, los métodos usados durante el desarrollo de la investigación fueron; análisissíntesis; inductivo-deductivo; histórico-lógico, y comparativo.

Análisis- síntesis. Se permitió conocer, comprender y aplicar, sobre la base de la descomposición de la variable dependiente (atención al cliente) y la variable independiente (calidad del servicio) en sus partes.

Inductivo-deductivo. El inductivo ayudó principalmente a no perder de vista las variables con las que se trabajó, relacionándolas con los aspectos generales y el deductivo para partir de conceptos generales que nos proporcionó, el marketing y áreas relacionadas teóricamente fundamentadas para llegar al tema en específico que es la atención al cliente.

Histórico-lógico. Permitió el conocimiento de la trayectoria y antecedentes generados en la heladería "El Estudiante".

Comparativo. Al establecer similitudes y disimilitudes entre los resultados obtenidos de las herramientas de recolección de datos en cuanto a la atención al cliente.

Las técnicas e instrumentos utilizados fueron la observación, la entrevista, y la encuesta.

Observación. El instrumento utilizado fue una guía prediseñada, el cual permitió ver el comportamiento de los clientes que acuden al establecimiento y del personal de la heladería "El Estudiante". También se analizó, el desarrollo de las actividades, la situación actual de la atención al cliente que se brinda y la calidad del servicio que se presenta con sus principales fallas.

Entrevista. El instrumento utilizado fue una guía de preguntas, que se le realizó al gerente propietario de la heladería "El Estudiante", el cual, ayudo a recabar información necesaria sobre la atención al cliente que se tiene en la empresa.

Encuesta. El instrumento utilizado fue, el cuestionario, el cual permitió entender mejor el comportamiento y las preferencias de los involucrados con respecto a la atención al cliente que se tiene en la actualidad.

La población, que se tomó en cuenta para el estudio estuvo comprendida por clientes actuales de la heladería "El Estudiante", entre hombres y mujeres cuya edad oscila de 14 a 50 años, que residen en el distrito urbano de la ciudad de Potosí y consumen en la heladería. Por la información proporcionada en la administración de la empresa en base al control que se lleva a cabo por el número de conos y canastillas de helados que son utilizados, se 
estima que en promedio ingresan 70 personas al día, lo que equivale a 2.100 clientes al mes. Para establecer la muestra se aplicó el procedimiento para poblaciones finitas en el que $n$ es igual a 325.

Cabe destacar, el muestreo que se empleó en la investigación fue el Muestreo probabilístico Aleatorio Simple (MAS), que significa que cualquier persona tiene la misma posibilidad de ser encuestada.

\section{RESULTADOS Y DISCUSIÓN}

\section{Dimensión 1. Actividades}

Esta dimensión fue dirigida al conjunto de actividades, acciones, operaciones y tareas que se realizan en la empresa, todas estas encaminadas a que se brinde una atención al cliente eficiente, es por ello que se muestra el análisis e interpretación de los diferentes instrumentos que se aplicaron para determinar cuál es la situación actual que se presenta en la heladería "El Estudiante" con respecto a esta dimensión, estas actividades realizadas en la empresa son sobre todo ejecutadas por el personal quienes tienen el contacto directo con el cliente. (Figura 1).

\section{f) El personal de la heladeria tiene la} capacidad para resolver los problemas de los dientes de unamanera efectiva

e) El tiempo de entrega de mi pedido fue oportuno

d) El personal de la heladeria se encuentra capacitado en cuanto a

atención al cliente

c) El personal de la heladería usó un lenguaje corporal adecuado al dirigirse a mi persona

b) El personal de la heladeria expresósus palabras de forma educada al atender

a) Al ingresar a la heladeria recibi un saludo cordial

- Totalmente de acuerdo

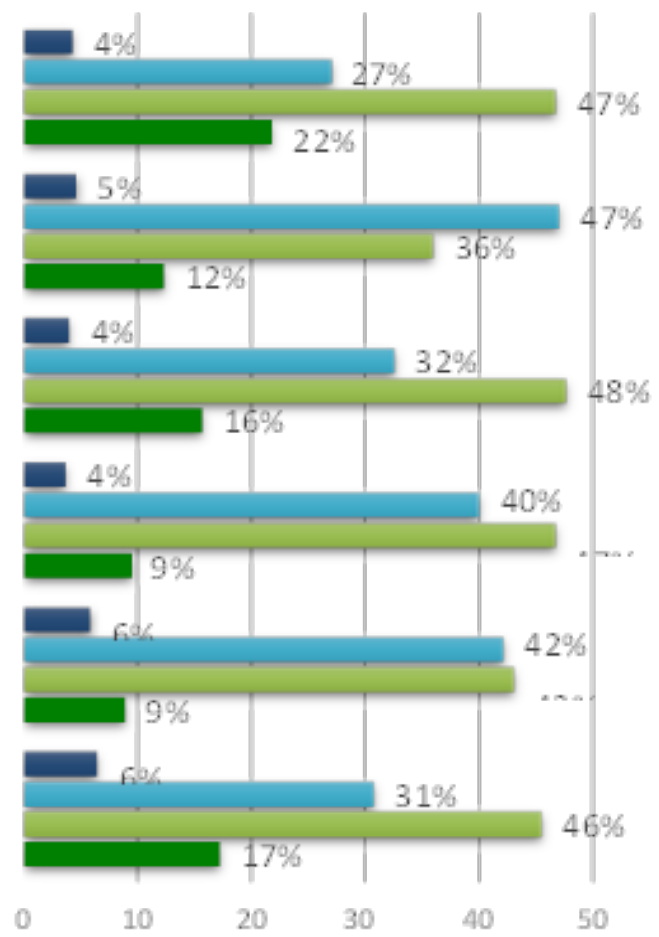

60

Figura 1. Actividades de atención al cliente que realiza el personal. 
Entre los elementos que llama la atención en la Figura 1 con relación al problema de atención al cliente se identifica que, el $46 \%$ de los encuestados se encuentra en desacuerdo en cuanto a si el personal brinda un saludo cordial al cliente cuando este ingresa a la heladería. También se identifica que el personal no se comunica de una manera asertiva, ya que, no expresa sus palabras de forma educada al dirigirse al cliente con una percepción del $43 \%$ que se encuentra en desacuerdo y con un lenguaje corporal inadecuado expresado en $47 \%$ de los resultados. En lo que respecta a la capacitación en atención al cliente con la que cuenta el personal, los encuestados opinaron que el $16 \%$ se encuentra totalmente en desacuerdo, el $48 \%$ está en desacuerdo. Otro dato destacable, es que el $22 \%$ se encuentra totalmente en desacuerdo, el $47 \%$ está en desacuerdo en cuanto a la capacidad del personal por resolver los problemas de los clientes de una manera efectiva. Estos resultados evidenciaron que se percibe falencias en las actividades que se realizan en la atención al cliente.

En este sentido, se puedo evidenciar que, el personal no brinda un saludo cordial cuando el cliente ingresa al establecimiento para realizar una compra, dejando de lado una primera buena atención que logre capturarlo de entrada, considerando esto como un elemento causal para que no exista una buena atención al cliente en la empresa. Además, existen falencias en la comunicación verbal y no verbal, ya que, se evidencio una incongruencia entre las palabras y el lenguaje corporal, con el que el personal se dirige al cliente, afectando negativamente a la atención al cliente. También, se mostró que los clientes al paso son atendidos más rápidamente a diferencia de los que permanecen en el local, sin embargo, el hecho perjudicial para la atención al cliente es debido a que el tiempo de entrega oportuno se ve afectado por el número de personas que existe en el momento de la atención generando molestias en el cliente. Y existe, la incapacidad por parte del personal para resolver problemas o atender una queja, siendo esto una causa que incide en la mala la atención al cliente, puesto que no se genera una solución, siendo que la empresa podría convertir los reclamos en oportunidades de negocio, aportándoles soluciones a los clientes.

\section{Dimensión 2. Necesidades}

Esta dimensión fue dirigida a la necesidad como estado de carencia física o mental que presentó el cliente ante el estado real, es decir, un momento de verdad, y el estado deseado, que supone al producto o servicio que necesita para su bienestar o confort. Es así que, en la atención al cliente las necesidades del cliente deben ser identificadas para posteriormente poder satisfacerlas. (Gráfico 1). 


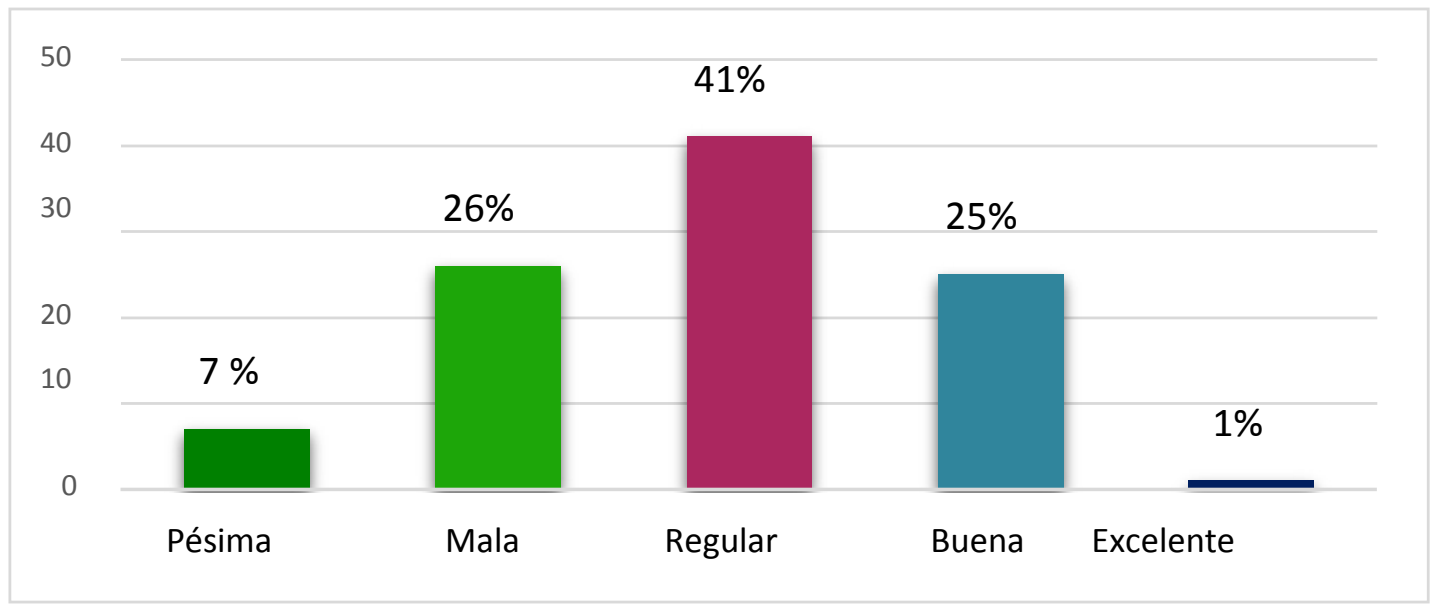

Gráfico 1. Cómo se adelanta la empresa a identificar lo que requiere el cliente.

Se muestra en el Gráfico 1, que el $7 \%$ de los clientes que participaron en la encuesta opina que la manera en que la heladería se adelanta a identificar lo que requiere el cliente es pésima, el $26 \%$ opina que es mala, el $41 \%$ dice que es regular, el $25 \%$ opina que es buena y solo el $1 \%$ considera que es excelente.

En los resultados se evidenció que, existe una mayor inclinación hacia el lado negativo, por lo que se deduce que la heladería no se adelanta a identificar lo que el cliente necesita, siendo desfavorable para la atención al cliente.

En cuanto a la solitud de información del producto antes de realizar el pedido por parte del cliente, se muestra en el Gráfico 2.

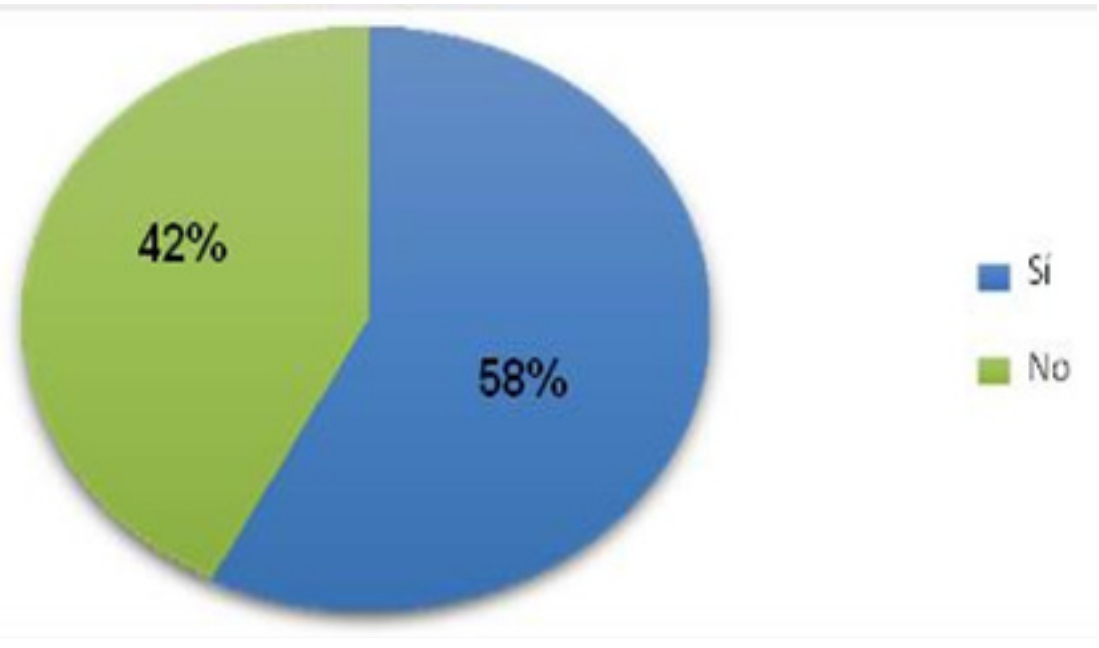

Gráfico 2. Clientes que pidieron información del producto. 
En el Gráfico 2, se muestra que, el $58 \%$ de los clientes pidió información del producto antes de consumirlo, mientras que el $42 \%$ de los clientes no lo hizo.

En este sentido, los resultados evidenciaron que, los clientes actuales de la heladería "El Estudiante" en un mayor porcentaje suelen pedir información del producto antes de realizar su pedido para adquirirlo, esta información puede consistir en varias opciones tales como sabores, tipo de producto, características del producto, tiempo de preparación, precio, etc., que el cliente necesita conocer.

Seguido, se muestra en el siguiente gráfico, la capacidad que tiene el personal en brindar dicha información a los clientes.

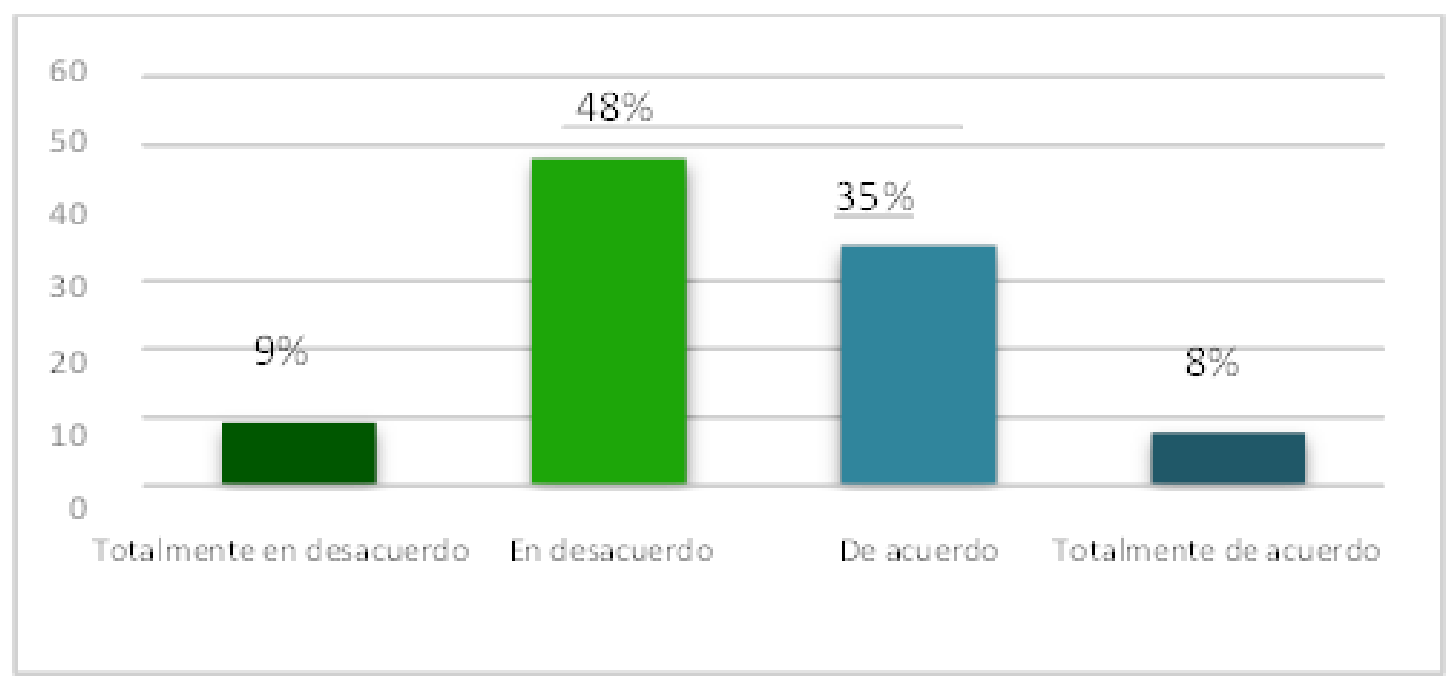

Gráfico 3. Capacidad del personal en brindar información.

En cuanto a la capacidad del personal en brindar información requerida del producto al cliente el Gráfico 3, muestra que el $9 \%$ se encuentra totalmente en desacuerdo, el $48 \%$ está en desacuerdo, el 35\% respondió de acuerdo y el $8 \%$ está totalmente de acuerdo.

Con lo anterior se evidencio que cuando se trata del producto, la heladería "El Estudiante" desempeña su trabajo de forma eficiente, todo esto para atender los requerimientos que presenta el cliente. Se reconoció, por lo tanto, el lado positivo de atención al cliente en la empresa al momento de preparar correctamente el producto que es ordenado por el cliente.

\section{Dimensión 3. Clientes}

Para continuar con la caracterización de la situación actual de la heladería "El Estudiante" se tomó en cuenta en esta dimensión al cliente Tal como se muestra en el Gráfico 4 individuo que teniendo la opción de recurrir a la empresa para adquirir un producto evalúa cómo la atención al cliente que recibe logra afectar su comportamiento, es por ello que se tuvo los siguientes resultados: 


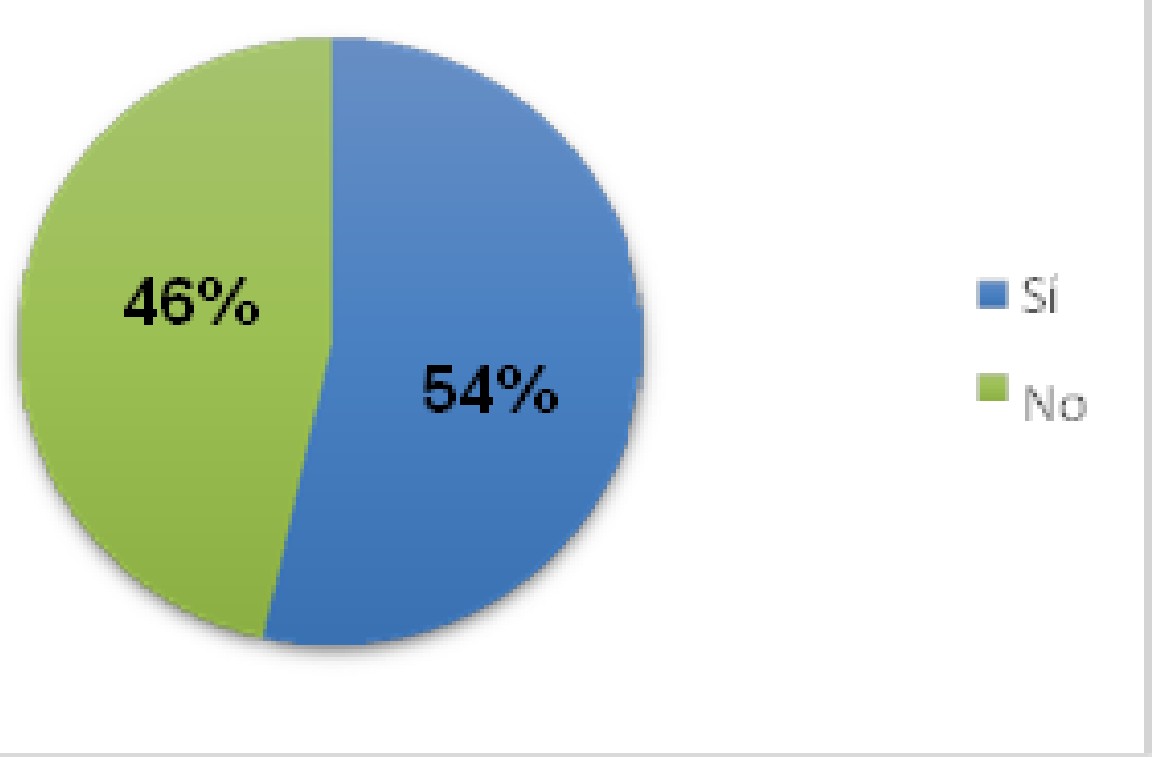

Gráfico 4. La atención al cliente afecta en la decisión de compra del cliente.

En el Gráfico 4, se muestra que, el $46 \%$ dijo que la atención que recibieron No afectó su decisión de compra y el $54 \%$ dijo que Sí le afectó.

Estos resultados evidenciaron que, más de la mitad de los encuestados, se vieron afectados por la atención que recibieron en la heladería "El Estudiante" y esto incurre en su decisión de compra, se dedujo por lo tanto que, la atención al cliente afecta directamente en el comportamiento de compra del cliente, si esta es buena de forma positiva y si esta es mala entonces afecta negativamente.

\section{Dimensión 4. Expectativas}

En esta dimensión se trató de reconocer si la atención al cliente que se brinda en la heladería logra cumplir las expectativas de los clientes. A continuación, se muestran los resultados con relación a esta dimensión. (Gráfico 5). 


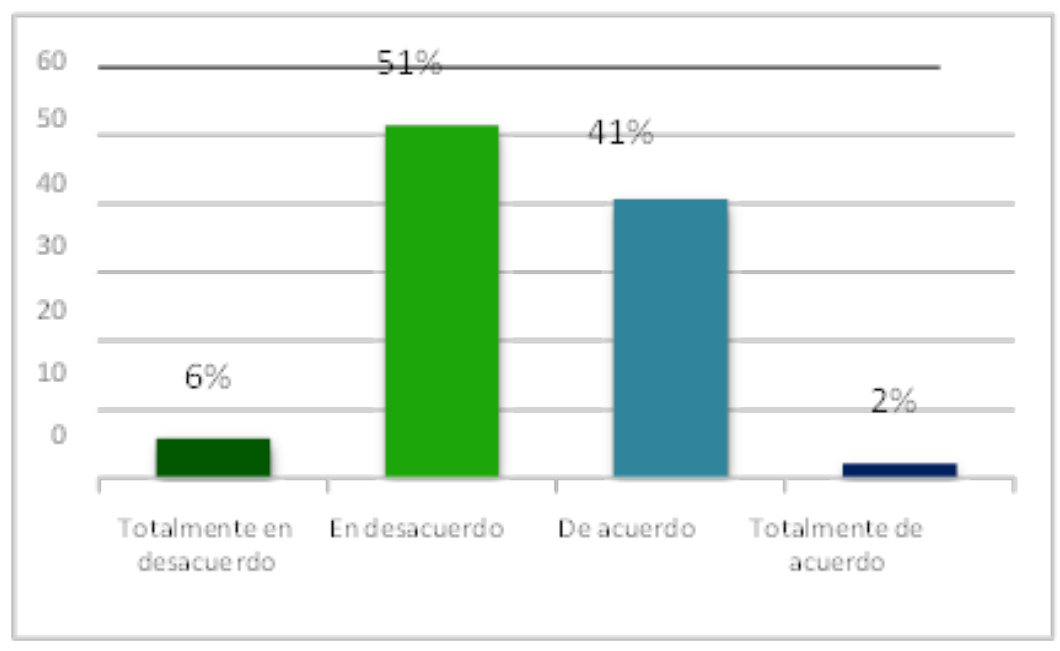

Gráfico 5. Paciencia del personal al tomar el pedido

En el Gráfico anterior, se muestra que, el $6 \%$ de los encuestados se encuentra totalmente en desacuerdo en que el personal fue paciente a la hora de tomar su pedido, el 51\% está en desacuerdo, el $41 \%$ de acuerdo y solo el $2 \%$ se encuentran totalmente de acuerdo. (Gráfico 5).

De esta forma, se evidenció que, la dedicación de tiempo a cada cliente mostró deficiencia, el personal de la heladería no es paciente a la hora de tomar el pedido al cliente, mostrándose así desventaja en la problemática de atención al cliente en la empresa, puesto que la dedicación de tiempo a cada cliente a través de la paciencia al momento de atenderlo es la clave para que este se sienta cómodo y especial.

En el Gráfico 6, se muestra la calificación del 1 al 5, siendo 1 que el cliente no se sintió a gusto y 5 que el cliente se sintió muy a gusto, en cuanto al impacto de la permanencia del cliente en la heladería "El Estudiante" se tiene que, el 3\% de los clientes calificó 1, el 22\% aprecia 2 , el $35 \%$ califica 3 , el $25 \%$ valora 4 y el $15 \%$ se sintió muy a gusto calificando 5 .

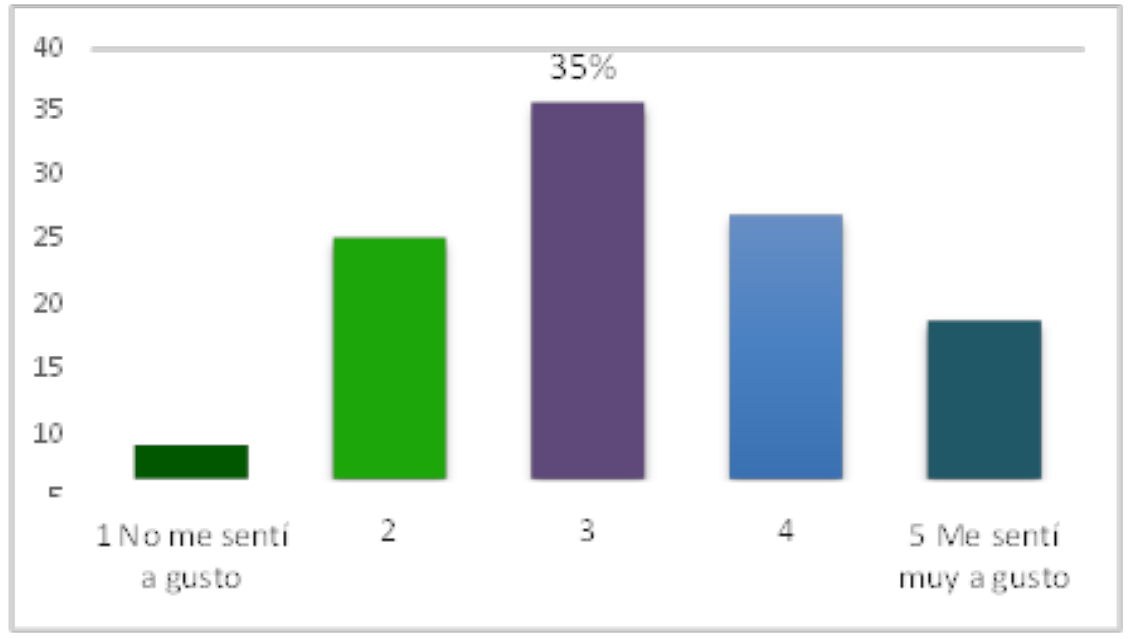

Gráfico 6. Calificación de impacto de la permanencia en la heladería. 
Los resultados demostraron que los clientes actuales en su mayoría suelen sentirse a gusto y muy a gusto durante su permanencia en la heladería, siendo este resultado un poco contradictorio con las anteriores preguntas de la misma dimensión, esto se debe a que como se dijo anteriormente las expectativas de los clientes resultan ser diferentes en cada uno de ellos, por lo tanto, la atención al cliente que reciben les genera también un impacto diferente.

\section{Dimensión 5. Satisfacción}

Esta dimensión trato sobre cómo la satisfacción del cliente se crea, incrementa o disminuye con la atención que recibe el cliente.

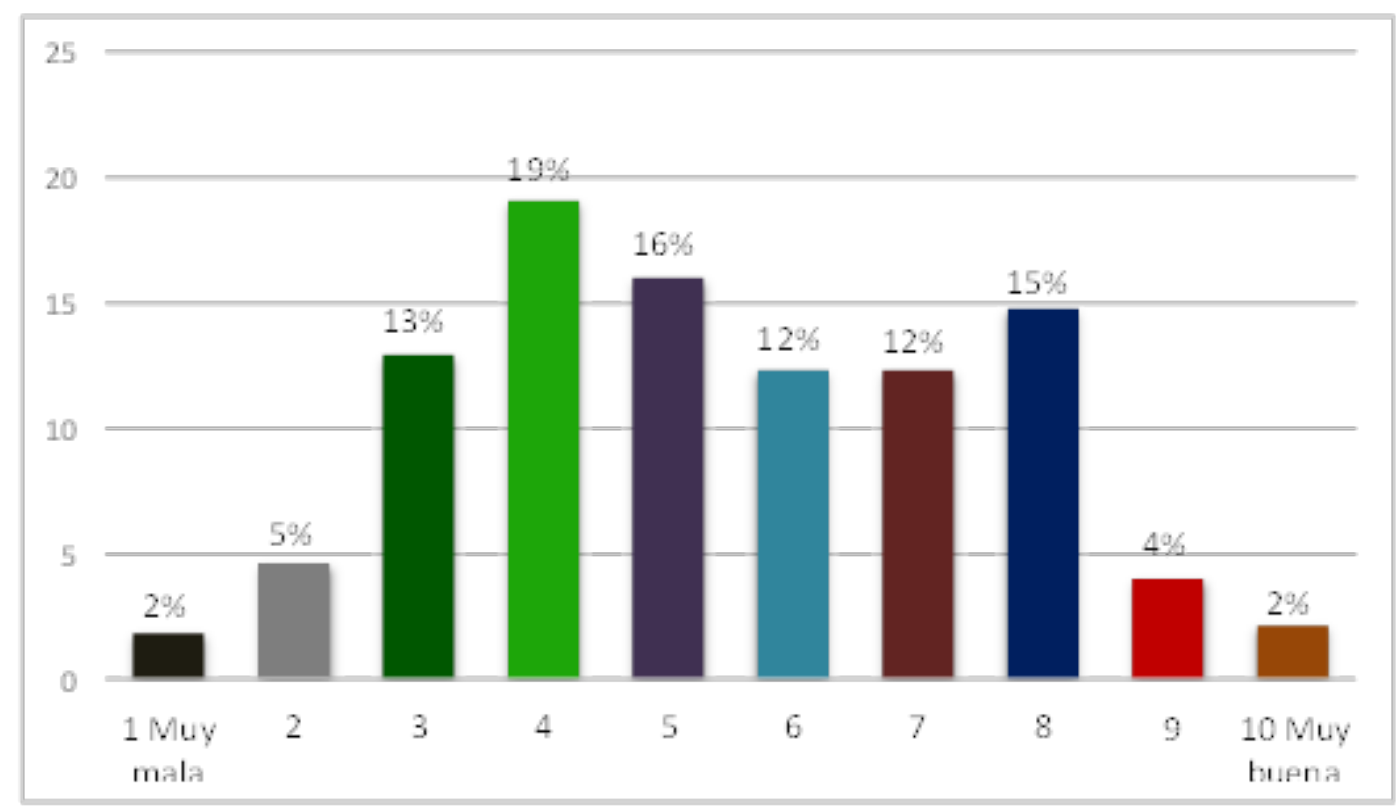

Gráfico 7. Calificación de la atención al cliente en la heladería El Estudiante del 1 al 10.

Se muestra en el Gráfico 7, la calificación del 1 al 10 sobre la atención al cliente de la heladería "El Estudiante", en donde 1 es muy mala y 10 muy buena, se tiene que alguna de las principales calificaciones fue; el 13\% califica 3, el 19\% juzga 4, el 16\% la valora como 5 , el $12 \%$ califica tanto 6 como 7 y el $15 \%$ califica 8 .

Con los resultados se evidenció que, existe una tendencia hacia el lado negativo en la calificación de la atención al cliente por parte de los encuestados, por lo que se deduce que los clientes no se encuentran muy satisfechos por el servicio que recibieron en la heladería y no se generó en ellos una gran experiencia de compra que los haya hecho sentir realmente especiales, es así que se sigue demostrando que existen falencias en la problemática planteada de atención al cliente. 
En este orden de ideas, se vio necesario la elaboración de una propuesta para darle solución a la problemática planteada en este estudio. Por ende, se desarrolla los aspectos más resaltantes de dicha propuesta.

\section{Propuesta sobre estrategia de calidad del servicio para mejorar la atención al cliente en la heladería "El Estudiante"}

Existe la necesidad en la ciudad de Potosí de que las heladerías o cualquier otro establecimiento comercial puedan brindar una buena atención al cliente, es así que a través de la calidad del servicio la heladería El Estudiante podrá mejorar la atención al cliente, no sólo para los clientes actuales, sino también para atraer clientes potenciales y cautivar a los clientes accidentales.

Con la calidad del servicio no solo se logrará satisfacer las necesidades del cliente, sino que se superará sus expectativas ofreciendo un servicio confiable, seguro, flexible, empático y un lugar cómodo y agradable.

En este sentido, la propuesta tiene por objetivo, plantear un conjunto de acciones sobre calidad del servicio para mejorar la atención al cliente en la heladería "El Estudiante" de la ciudad de Potosí.

El desarrollo de la propuesta está basado en las cinco dimensiones de la calidad del servicio que son la confiabilidad, sensibilidad, seguridad, empatía y tangibles como se mostró en la estructura.

Confiabilidad. Cumplir las promesas es muy importante para los clientes, a partir de esto en la empresa se debe tratar de cumplir con todo lo que se dice y promete:

Sensibilidad. Estar dispuesto a ayudar al cliente, tratar de solucionar, preguntas, quejas y problemas.

Seguridad. Inspirar credibilidad y confianza, Infundir confianza al cliente, estar a salvo de peligros y dudas, cortesía de los empleados y tener conocimiento del producto. Para que la heladería inspire credibilidad y confianza el personal debe tener el conocimiento pleno de los productos que está ofertando y de todo el movimiento que se tiene en la empresa para que se brinde seguridad al cliente.

Empatía. Tratar a los clientes como individuos. Es importante para este tipo de dimensión que la estrategia esté dirigida a los empleados que trabajan dentro de la heladería "El Estudiante" quienes tienen el contacto directo con el cliente. Atención personalizada y cuidadosa, hacer sentir especial e importante al cliente.

Tangibles. Representar al servicio físicamente. La presentación de los productos, el decorado, los muebles y todas las cosas materiales que se encuentran dentro de la heladería tendrán que estar perfectamente presentables.

Adicional, es importante la presencia personal, ya que, el personal debe mostrar una imagen pulcra por lo que su atuendo debe ser correcto, pero debe permitirle moverse con soltura y dignidad. Para ellos se propone para el personal uniformes que muestren al cliente un grado de profesionalidad en los empleados. 
Los uniformes serán realizados a la talla de cada empleado y este será personalizado ya que contará con el nombre de cada uno, consiste en una camisa y también en una chaqueta para que el personal pueda alternar su uso. Los uniformes deberán ser usados todos los días para que el empleado sienta su categoría profesional incrementada, sin embargo, se dará la libertad a los empleados que puedan usar cualquier prenda los días sábados y domingos sin dejar de lado la pulcritud.

Los colores del uniforme serán naranja y verde por la significación que estos tendrán para la heladería.

\section{CONCLUSIONES}

$\mathrm{P}$ artiendo del objetivo de la investigación y dando cumplimiento al mismo, se concluye; que la atención al cliente es un elemento de vital importancia en la empresa ya que como conjunto de actividades busca que la empresa identifique y satisfaga las necesidades de los clientes a través de la comunicación e interacción directa con ellos y cumplir con sus expectativas. A su vez, la calidad del servicio se define como la percepción que tiene un cliente a cerca del desempeño de la empresa en la prestación de sus servicios y sus expectativas, relacionadas con el cumplimiento de las especificaciones y es analizada en sus cincos dimensiones; confiabilidad, sensibilidad, seguridad, empatía y tangibles.

Además, existen varias falencias que son causales para que no se cuente con una atención al cliente ideal en la empresa, comprobando que en la actualidad se tiene esta problemática. Lo más rescatable que tiene la empresa es el reconocimiento que tiene por el producto y no así por el servicio en atención al cliente.

Para finalizar, con la necesidad de mejorar la atención al cliente en la heladería "El Estudiante", se presenta una propuesta en base a los elementos de calidad del servicio, en la que se brinda herramientas y acciones que serán de gran ayuda para solucionar la problemática por la que atraviesa la empresa.

\section{REFERENCIAS}

Cultural S.A. (1999). Diccionario de Marketing / Cultural S.A. España

Horovitz, J. (1991). La calidad del servicio. A la conquista del cliente. Editorial McGraw-Hill; España, pp. 2-3

Kotler, P. (2003). Los 80 Conceptos esenciales del Marketing de la A la Z. México: Pearson Educación S.A.

Kotler, P., y Armstrong, G. (2008). Fundamentos de marketing. ( $8^{\mathrm{a}}$. ed.). Naucalpan de Juárez. Estado de México: Pearson Prentice Hall

Kotler, P., y Keller, K. (2012). Dirección de Marketing. (14a. Ed.). Naucalpan de Juárez. Estado de México: Pearson Educación

López, M. (2013). Importancia de la calidad del servicio al cliente. El buzón de Pacioli, 82, p.4
Palomo, M. (2014). Atención al cliente. (1a. Ed.). Madrid, España: Ediciones Paraninfo SA

Paz, R. (2007). Atención al cliente. Guía práctica de técnicas y estrategias. (1a. ed.). España: Ideas propias Editorial

Tigani, D. (2006). Excelencia en Servicio. Editorial: Liderazgo 21, p.70

Thompson, I. (2008). Marketing de Servicios. Marketing-Free. Recuperado de: http://www. marketing-free.com/glosario/marketingservicios.html

Zeithaml, V.; Bitner, M., y Gremler, D. (2009). Marketing de Servicios. (5a. ed.). México: McGraw-Hill / Interamericana Editores, S.A. de C.V. 